\title{
APERTURA COMERCIAL Y DESIGUALDAD SALARIAL EN MÉXICO: UN ANÁLISIS REGIONAL PARA LOS AÑOS 1992 Y $2014^{*}$
}

\author{
Recibido: 13 de diciembre de 2016 - Aprobado: 04 de septiembre de 2017 \\ DOI: $10.22395 /$ seec.v20n45a4 \\ David Castro Lugo* \\ Albany Aguilera Fernández ${ }^{* * *}$
}

\section{RESUMEN}

Este artículo evalúa el efecto generado por el proceso de apertura comercial sobre la estructura del mercado de trabajo y la desigualdad salarial en México, para los años 1992 y 2014, utilizando información de la Encuesta Nacional de Empleo Urbano y Encuesta Nacional de Ocupación y Empleo. El ejercicio de microsimulación a escala regional indica que en ausencia de apertura comercial, la disparidad sería inferior a lo observado en 2014. La estructura de empleo por tipo de calificación y la remuneración fueron las principales fuentes de cambio en la desigualdad laboral. En México, contrario a las predicciones teóricas, la apertura de la economía al comercio exterior no contribuyó a reducir las disparidades a largo plazo, aunque en 2014 la región mexicana con mayor exposición al comercio exterior presenta menor desigualdad (0.357) en relación con la menos expuesta (0.393), diferencia que es superior a la observada en 1992 (0.397 vs 0.410).

\section{PALABRAS CLAVE}

Apertura comercial; comercio exterior; mercado de trabajo; desigualdad salarial; microsimulaciones; México.

\section{CLASIFICACIÓN JEL}

F31, J21, J31, R23

\section{CONTENIDO}

Introducción; 1. Desigualdad salarial y apertura comercial: teoría y evidencia; 2. Metodología, datos y regionalización; 3 . Tendencias del mercado de trabajo regional en México; 4. Desigualdad salarial en las regiones de México; 5. Conclusiones; Bibliografía.

\footnotetext{
Este artículo de investigación es producto de los resultados obtenidos en la tesis doctoral titulada "Apertura comercial en México y su efecto sobre el mercado laboral y las disparidades salariales: una aplicación regional", desarrollada por Albany Aguilera Fernández, bajo la dirección de David Castro Lugo para el programa de Doctorado en Economía Regional, generación 2012-2016, Universidad Autónoma de Coahuila y con el apoyo del Consejo Nacional de Ciencia y Tecnología (Conacyt) de México. Los autores agradecen los comentarios y observaciones de pares evaluadores anónimos.

“* Economista, Universidad Autónoma de Sinaloa, México. Maestro en Economía Aplicada, Colegio de la Frontera Norte, Tijuana, México. Doctor en Economía Aplicada, Universidad Autónoma de Barcelona, España. Profesor e investigador, Centro de Investigaciones Socioeconómicas de la Universidad Autónoma de Coahuila, Saltillo, México. Dirección: Unidad Camporredondo edificio "S", CP 25080, Saltillo, Coahuila, México. Tel 52 (844)4121113 ext. 109. Correo electrónico: david.castro@uadec.edu.mx.

"** Contadora, Universidad Autónoma de Zacatecas, México. Maestra en Economía Regional, Centro de Investigaciones Socioeconómicas, Saltillo, México. Doctora en Economía Regional, Centro de Investigaciones Socioeconómicas, Saltillo, México. Profesora de Tiempo Completo, Facultad de Economía y Mercadotecnia de la Universidad Autónoma de Coahuila, Torreón, México. Dirección: Blvd. Torreón-Matamoros Km 7.5 Ciudad Universitaria, CP 27276, Torreón, Coahuila, México, Tel (871) 716-77-84. Correo electrónico: albany.aguilera@uadec.edu.mx.
} 


\section{TRADE OPENING AND WAGE INEQUALITY IN MEXICO: A REGIONAL ANALYSIS FOR 1992 AND 2014}

\section{ABSTRACT}

This paper assesses the effect generated by the trade opening process on the structure of labor market and wage inequality in Mexico for the years 1992 and 2014, using information from the National Urban Employment Survey and the National Employment and Occupation Survey. The micro-simulation exercise at regional level indicates that in the absence of trade liberalization, the disparity would be lower than in 2014. The structure of employment by qualification type and remuneration were the main sources of change in labor inequality. In Mexico, contrary to theoretical predictions, the opening up of the economy to foreign trade did not contribute to reduce long-term disparities, although in 2014 the Mexican region with greater exposure to foreign trade presents less inequality $(0.357)$ in relation to the least exposed $(0.393)$, a difference that is higher than the observed in 1992 (0.397 vs. 0.410).

\section{KEYWORDS}

Trade aoerture; foreign trade; labor market; wage inequality; micro-simulations; Mexico.

\section{JEL CLASSIFICATION}

F31, J21, J31, R23

\section{CONTENT}

Introduction; 1. Wage inequality and trade openness: theory and evidence; 2 . Methodology, data and regionalization; 3. Regional labor market trends in Mexico; 4. Wage inequality in the regions of Mexico; 5. Conclusions; Bibliography.

\section{ABERTURA COMERCIAL E DESIGUALDADE SALARIAL NO MÉXICO: UMA ANÁLISE REGIONAL PARA OS ANOS $1992 \mathrm{E} 2014$ RESUMO}

Este artigo avalia o efeito gerado pelo processo de abertura comercial sobre a estrutura do mercado de trabalho e a desigualdade salarial no México, para os anos 1992 e 2014, utilizando informação da Pesquisa Nacional de Emprego Urbano e da Pesquisa Nacional de Ocupação e Emprego. O exercício de microssimulação no âmbito regional indica que, em ausência de abertura comercial, a disparidade seria inferior ao observado em 2014. A estrutura de emprego por tipo de qualificação e remuneração foram as principais fontes de mudanças na desigualdade no trabalho. No México, contrariamente às predições teóricas, a abertura da economia ao comércio exterior não contribuiu para reduzir as disparidades em longo prazo, embora, em 2014, a região mexicana com maior exposição ao comércio exterior tenha apresentado menor desigualdade $(0,357)$ em comparação com a menos exposta $(0,393)$, diferença que é superior à observada em $1992(0,397$ versus 0,410).

\section{PALAVRAS-CHAVE}

Abertura comercial; comércio exterior; mercado de trabalho; desigualdade salarial; microssimulações; México.

\section{CLASSIFICAÇÃO JEL}

F31, J21, J31, R23

\section{CONTEÚDO}

Introdução; 1. Desigualdade salarial e abertura comercial: teoria e evidência; 2. Metodologia, dados e regionalização; 3 . Tendências do mercado de trabalho regional no México;

4. Desigualdade salarial nas regiões do México; 5. Conclusões; Bibliografia. 


\section{INTRODUCCIÓN}

Tanto en economías desarrolladas como en las emergentes y en desarrollo, los salarios representan la principal fuente de ingresos de los hogares. De acuerdo con la OIT (2015), en el año 2012 los salarios constituyeron entre el 70 y 80\% del total de la renta familiar en las economías desarrolladas, mientras que en las economías emergentes y en desarrollo osciló entre un 50 y 60\%. De ahí que, la desigualdad salarial es un tema relevante, al considerarse un problema persistente que ha caracterizado el mercado de trabajo con efectos adversos sobre la cohesión social, el bienestar laboral y el crecimiento económico a mediano y largo plazo (OCDE, 2015).

Uno de los debates más significativos en relación con dicho fenómeno estriba en si a raíz de la apertura comercial la mano de obra menos calificada es beneficiada. La tendencia observada en la evolución de los ingresos en economías abiertas ha llevado a cuestionar la teoría neoclásica del comercio internacional HeckscherOhlin y el teorema Stolper-Samuelson. En el caso de México, y considerando este enfoque, se esperaba que con el proceso de apertura de la economía incrementaran los salarios relativos para aquellos que representan el factor más abundante, y con ello reducir la desigualdad entre trabajadores con distintas calificaciones.

La evidencia empírica actual sugiere que el efecto del comercio exterior sobre los salarios relativos es ambiguo a corto plazo, por lo que no se han validado a plenitud las predicciones teóricas. Como principal argumento para el incumplimiento de esta hipótesis Cañonero y Werner (2002), Burgos y Mungaray (2008) y Pérez (2014) expresan que la temporalidad de los estudios realizados es insuficiente para capturar los efectos económicos planteados. Por otra parte, Hanson (2007), Chiquiar (2008), Garduño y Baylis (2012), Tello y Ramos (2012) y De la Mora (2015) destacan la importancia de la diferenciación territorial en la evolución de los perfiles salariales específicos de cada Estado o unidad administrativa subnacional, puesto que la aceleración de los procesos de globalización incide de forma heterogénea entre las distintas regiones del país, donde no todas han logrado integrarse con éxito a los mercados internacionales.

En este sentido, el objetivo del artículo es evaluar el efecto del proceso de apertura comercial sobre la estructura del mercado de trabajo y la desigualdad salarial en México, con información de los años 1992 y 2014. Los datos utilizados corresponden a la Encuesta Nacional de Empleo Urbano (ENEU) y Encuesta Nacional de Ocupación y Empleo (ENOE). Se exploran indicadores del mercado de trabajo y se aplica la metodología de microsimulaciones, técnica que permite identificar qué parte de los cambios observados en la desigualdad del ingreso laboral es asociada 
a la liberalización comercial, así como aislar los determinantes por modificaciones en los parámetros del mercado de trabajo.

Dentro de los principales hallazgos se destaca el crecimiento de las tasas de desempleo en todas las regiones y por sexo; igualmente, se aprecia un aumento de la participación laboral de trabajadores semicalificados y calificados, así como una caída generalizada de los salarios reales en todas las regiones y niveles de calificación. El ejercicio de microsimulación sugiere que la apertura comercial no contribuyó a reducir las disparidades salariales a largo plazo, ya que, de haberse mantenido la estructura del mercado laboral previo al proceso de liberalización económica, los niveles de desigualdad serían inferiores a los observados en 2014. La estructura de empleo por tipo de calificación y la remuneración de los mismos fueron las principales fuentes de cambio en la desigualdad laboral.

El resto del documento está estructurado como sigue: inicia con las bases teóricas que rigen la relación entre apertura comercial y desigualdad salarial, así como una revisión de la literatura en torno al tema. A continuación, se detalla la metodología de microsimulación, los datos y regionalización a utilizar. El tercer apartado muestra las características de los mercados de trabajo regionales, y los resultados del efecto de la liberalización comercial sobre la distribución del ingreso se presentan en el apartado cuatro. Se finaliza con las conclusiones de la investigación.

\section{DESIGUALDAD SALARIAL Y APERTURA COMERCIAL: TEORÍA Y EVIDENCIA}

Las disparidades salariales pueden clasificarse como un problema persistente que ha caracterizado al mercado laboral de diversos países. Para comprender la inequidad en los ingresos, Castro y Huesca (2007) ofrecen una gama de posibles explicaciones relacionadas con alteraciones en tres factores: demanda relativa de mano de obra, oferta relativa e instituciones del mercado de trabajo. En el caso mexicano, Cortez (2004) y Luyando (2013) enfatizan que las modificaciones de la desigualdad salarial responden, en gran medida, a transformaciones en los componentes de la demanda relativa de mano de obra, de la cual destacan dos perspectivas.

La primera refiere a la hipótesis del cambio tecnológico sesgado por calificación, orientada al capital y el conocimiento. De acuerdo con lo que teorizan expertos en esta línea de estudio, los avances tecnológicos, traducidos en mayor productividad e innovaciones, favorecen la demanda y el salario relativo de trabajadores calificados, propiciando un aumento del diferencial salarial (Acemoglu, 1998). A la luz de la evidencia para México, se ha encontrado una correspondencia entre las diferencias del ingreso laboral y las brechas tecnológicas (Camberos, Huesca y Castro, 2013). 
La segunda perspectiva se sustenta en la teoría convencional del comercio internacional Heckscher-Ohlin y la propuesta de Stolper y Samuelson (1941), de la cual se deriva que con la apertura comercial los precios y salarios relativos tenderán a nivelarse, ya que las naciones se especializarán y exportarán aquellos bienes en los cuales poseen ventaja frente al resto de los países e importarán aquellos en los que tienen desventajas, lo que llevará a un incremento de la demanda y el salario relativo del factor de producción abundante, en relación con el factor escaso.

Bajo cualquier enfoque, México resulta un adecuado caso de estudio por su proceso de liberalización comercial, que se distingue por dos acontecimientos principales. El primero ocurrió en 1986 con la integración al Acuerdo General sobre Aranceles Aduaneros y Comercio (GATT por sus siglas en inglés), caracterizado por una disminución significativa de los aranceles, permisos de importación y una reducción gradual de las restricciones a la inversión extranjera. El segundo evento se identifica con la firma del Tratado de Libre Comercio de América de Norte (TLCAN) entre Estados Unidos de América, México y Canadá, que entró en vigor el 1 de enero de 1994 y con el cual institucionalizó la estrategia de liberalización comercial de México; a partir de este año, el grado de apertura comercial alcanzó niveles superiores al 50\%. A continuación, el país se incorporó a la Organización para la Cooperación y el Desarrollo Económicos (OCDE) y la Organización Mundial del Comercio (OMC), y firmó otros acuerdos importantes con países de América Latina, la Unión Europea y Japón.

Blecker (2010) sostiene que, con la liberalización comercial y la firma del TLCAN, se esperaba atraer mayor inversión extranjera directa (IED) y fomentar el crecimiento económico, mediante el aumento de las exportaciones intensivas en el uso del factor abundante y destinadas al mercado estadounidense, es decir, la mano de obra menos calificada. En este contexto, a partir de la década de los noventa surgieron diversos estudios empíricos para México que examinan la relación entre el comercio y las diferencias salariales, y encuentran resultados inconclusos con respecto a si la relación ha sido positiva o negativa en términos de disparidad salarial.

En esta línea de análisis Robertson (2004), Airola y Juhn (2005), Borraz y LópezCórdova (2007), Chiquiar (2008) y Garduño y Baylis (2012) proporcionan evidencia de una reducción de la desigualdad salarial en los mercados laborales mexicanos a raíz de la política comercial. Dichos autores llegan a conclusiones similares: que posterior a la entrada en vigor del TLCAN en 1994, se observaron cambios en los precios relativos de los factores a favor de la mano de obra no calificada; no obstante, Ros y Bouillon (2002), Ramírez (2004), Burgos y Mungaray (2008), Luyando (2013) y López (2015) sugieren que la globalización ha sesgado la demanda en favor 
de la mano de obra calificada y en detrimento de la no calificada, por consiguiente, ha contribuido a una mayor desigualdad salarial entre trabajadores con distinto nivel de calificación.

Al respecto, se han ofrecido diversos argumentos que justifican por qué los efectos de la apertura comercial difirieren del pronóstico del teorema Stolper-Samuelson. El de mayor énfasis es planteado por Cañonero y Werner (2002) y Blecker (2010), quienes argumentan que el teorema debe considerarse en el largo plazo, lo que determinaría la respuesta de los precios de los factores a un cambio en los precios de los bienes; además, Hanson (2007), Chiquiar (2008) y De la Mora (2015) admiten la importancia de la dimensión espacial del análisis, ya que no se mantiene el mismo resultado cuando se estudia toda la economía en su conjunto, que cuando se examina a una escala regional, debido a que las regiones, sectores y empresas de México exhiben diferencias en cuanto al grado de exposición hacia los mercados internacionales, por lo que el impacto de la liberalización del comercio sobre los precios de los factores puede ser heterogéneo. Por dichas razones, surge el interés de analizar el impacto de la apertura comercial sobre la desigualdad salarial, considerando el ámbito regional.

\section{METODOLOGÍA, DATOS Y REGIONALIZACIÓN}

En esta sección se describe la metodología utilizada, los índices de desigualdad a estimar, las fuentes de información y la regionalización.

\subsection{Microsimulaciones}

En el análisis de desigualdad salarial se optó por utilizar la metodología de microsimulaciones, desarrollada por Almeida dos Reis y Paes de Barros (1991) y extendida por Ganuza, De Barros y Vos (2002). En evidencia precursora para México (Bouillon, 2000; Ros y Bouillon, 2002), la aproximación tradicional del método ha sido nacional y con información de hogares. De ahí que, el aporte de este estudio es adaptar la metodología y extenderla a escala regional, utilizar microdatos de individuos y unificar las bases de dos encuestas sobre fuerza laboral: la Encuesta Nacional de Empleo Urbano (ENUE) y la Encuesta Nacional de Ocupación y Empleo (ENOE).

La metodología considera el supuesto de que el mercado de trabajo es el canal de transmisión de los efectos de la apertura comercial sobre la desigualdad, por lo que se crean escenarios contrafactuales donde los parámetros del mercado laboral, "antes" a la ejecución de la política de liberalización comercial, son aplicados a la información de la encuesta de trabajo "después" de la liberalización, para simu- 
lar cuál habría sido la distribución del ingreso si los cambios en los parámetros no se hubieran implementado.

El análisis se conduce a través de la siguiente lógica: se asume que los agentes deciden participar o no; el mercado define si encuentran empleo; si han encontrado trabajo, el proceso de ajuste del mercado laboral determina en qué sector obtienen empleo, y sus decisiones de trabajar pueden estar influenciadas por la remuneración relativa que obtendrán (Ganuza, De Barros y Vos, 2002). En consecuencia, la estructura del mercado de trabajo en el año se expresa por las siguientes variables:

$$
\lambda=f(\mathrm{P}, \mathrm{U}, \mathrm{S}, \mathrm{W}, \mathrm{M})
$$

Donde $\mathrm{P}=\left[p_{j}\right]$ es la tasa de participación de la fuerza de trabajo de cada grupo de individuos del tipo $j$; $U=\left[u_{j}\right]$ es la tasa de desempleo de cada grupo de individuos del tipo j; la matriz $\mathrm{S}$ representa la estructura del empleo definida en términos de sector de actividad económica para los individuos de tipo $j$ en el segmento $k$; la matriz W indica la remuneración salarial (ingreso laboral de cada tipo de empleo en relación al ingreso laboral medio total) para los trabajadores del tipo $j$ en el segmento $k$, y la matriz $\mathrm{M}$ es la estructura de empleo en términos de calificación para cada grupo de trabajadores del tipo $j$. La población en edad de trabajar en el año $t$ se clasifica en tipos de individuos j sobre la base de género y nivel de calificación, mientras que la fuerza de trabajo ocupada se diferencia según el segmento $k$ delimitado por actividad económica.

Los ingresos de un individuo $i$ pueden variar como resultado de las condiciones del mercado de trabajo y características personales como género, edad, calificación, posición en el mercado de trabajo y otros atributos individuales representados por la variable $c$. Por lo tanto:

$$
y l_{i}^{*}=f\left(\lambda, c_{i}\right)
$$

De manera homóloga, $\lambda^{*}$ representa una estructura del mercado de trabajo contrafactual, expresado por:

$$
\lambda^{*}=f^{*}\left(\mathrm{P}^{*}, \mathrm{U}^{*}, \mathrm{~S}^{*}, \mathrm{~W}^{*}, \mathrm{M}^{*}\right)
$$

En la aplicación de la metodología de microsimulación, las estimaciones de las cinco variables integrantes del mercado de trabajo del año serán reemplazadas por aquellas del año, es decir, los efectos serán evaluados mediante la alteración de las variables P, U, S, W y M de forma individual o acumulativa (Ganuza, De Barros y Vos, 2002). 
La distribución del ingreso del individuo $(y l)$ en el año $t$ es una función de las variables del mercado de trabajo ya mencionadas, la distribución de los atributos individuales representados por $c$, y otros factores estructurales y/o coyunturales capturados por un parámetro $a$ :

$$
\mathrm{D}\left(y l_{i}\right)=\mathrm{D}(\mathrm{P}, \mathrm{U}, \mathrm{S}, \mathrm{W}, \mathrm{M}, c, a)=\mathrm{F}(\lambda, c, a)
$$

De manera similar, la distribución del ingreso del individuo $(y l)$ en el año $t^{*}$ es definido como:

$$
\mathrm{D}^{*}\left(y l_{i}\right)=\mathrm{F}\left(\lambda^{*}, c^{*}, a^{*}\right)
$$

En el supuesto de que los otros factores a son constantes, las modificaciones en los ingresos laborales entre dos años se pueden expresar como una función de cambios en las cinco variables del mercado de trabajo y en los atributos individuales (c):

$$
\Delta \mathrm{D}\left(y l_{i}\right)=\mathrm{D}\left(\mathrm{P}^{*}, \mathrm{U}^{*}, \mathrm{~S}^{*}, \mathrm{~W}^{*}, \mathrm{M}^{*}, \mathrm{c}^{*}\right)-\mathrm{D}(\mathrm{P}, \mathrm{U}, \mathrm{S}, \mathrm{W}, \mathrm{M}, \mathrm{c})
$$

Así, los valores de los parámetros antes de la liberalización (año inicial) serán los datos de la encuesta después de la liberalización (año final). El procedimiento para simular la trasformación en la estructura del mercado de trabajo se efectuará mediante cambios en cada parámetro de forma independiente, que seguirán el proceso probabilístico que se especifica en Ganuza, De Barros y Vos (2002, p. 83-86).

\subsection{Simulación de las medidas de desigualdad}

Una vez generada una nueva estructura del mercado de trabajo y distribución de ingreso en cada simulación, se puede aplicar cualquier medida de desigualdad. Los índices se calculan con los datos reales observados en el año base, y para los datos del año base cuando se imponga la estructura de la fuerza laboral del año contrafactual o simulado. En caso de que el valor simulado sea superior al observado, implica que la liberalización contribuyó a generar una reducción de la desigualdad. Si, por el contrario, el valor simulado es inferior al observado en el año final, significa que la liberalización produjo un aumento de la disparidad en el ingreso (Bouillon, 2000).

Como medida de desigualdad se estima el coeficiente de Gini, que oscila entre valores de 0 y 1 , donde cero corresponde a perfecta igualdad y uno implica total desigualdad. El cálculo se lleva a cabo mediante la siguiente formula:

$$
\mathrm{G}=\frac{1}{2 n^{2} \mu} \sum_{i=1}^{m} \sum_{j=1}^{m}\left|y_{i}-y_{j}\right|
$$


Dónde G es el coeficiente de Gini; $n$ es el tamaño de la población; $y_{i}, y_{j}$ el ingreso del i-ésimo o j-ésimo individuo; $\mu$ es el ingreso medio y $m$ es el número de ingresos distintos.

Adicionalmente, se estima el índice de Theil, herramienta analítica que permite desagregar en dos componentes para identificar la disparidad al interior de los grupos (within group) y entre grupos (between group). El valor mínimo que alcanza es cero, lo que denota equidad absoluta, en tanto que, su valor máximo no se encuentra acotado (Shorrocks, 1980). La fórmula general del componente intra se define por:

$$
\mathrm{T}_{w}=\sum_{j=1}^{k} \frac{n_{j} \mu_{j}}{n \mu} \mathrm{T}_{j}
$$

Donde $n$ es el total de observaciones; $n_{j}$ es el tamaño de la subpoblación $j$ ( $\mathrm{j}=$ $1,2, \ldots, \mathrm{k}) ; \mu$ es el ingreso medio y $\mu_{j}$ : es ingreso medio de la subpoblación $j$. El componente entre se obtiene con la expresión:

$$
\mathrm{T}_{6}=\sum_{i=1}^{n_{j}} \frac{n_{j}}{n} \frac{\mu_{j}}{\mu} \log \left(\frac{\mu_{j}}{\mu}\right)
$$

Donde es el total de observaciones; $n_{j}$ es el tamaño de la subpoblación $j$ ( $\mathrm{j}=$ $1,2, \ldots, \mathrm{k}) ; \mu$ es el ingreso medio y $\mu_{j}$ : es ingreso medio de la subpoblación $j(j=$ $1,2, \ldots, \mathrm{k})$.

\subsection{Datos}

Se optó por realizar un escenario a largo plazo en el que los parámetros del mercado de trabajo de 1992, considerado como año contrafactual y previo al TLCAN, serán aplicados a la información de la encuesta del 2014, nuestro año base y situación actual. Los años de análisis pueden considerarse comparables en términos de comportamiento macroeconómico, dado que presentan tasas de crecimiento positivas, aunque moderadas, 3,5 y $2,2 \%$, respectivamente.

Se recurrió a las bases de datos ENEU de 1992 y ENOE de 2014 que proporciona el Instituto Nacional de Estadística y Geografía (INEGI), y para obtener la representatividad de la población se aplicó el factor de expansión que contienen dichas encuestas. La variable de análisis es el salario real por hora, por lo que el salario mensual registrado en la ENEU fue deflactado con el Índice Nacional de Precios al Consumidor (INPC) correspondiente al mes de agosto de 2014. La muestra incluye a la población asalariada, tanto del sexo masculino como femenino que se encuentran en el rango de edad entre 14 y 65 años, que corresponde a la edad mínima para trabajar en México' y la edad legal de jubilación.

A partir de junio de 2014 se elevó a 15 años la edad mínima para trabajar en México. 
Se adoptaron grupos demográficos en función al nivel de calificación de la mano de obra con base en la clasificación de Aguilera y Castro (2017), quienes catalogaron a los trabajadores mediante el análisis de conglomerados jerárquico aglomerativo. De lo anterior se determinaron tres tipos de trabajadores: no calificados (NC) que tienen entre 0 y 9 años de escolaridad, semi calificados (SC) 10 y 14 años de escolaridad, y calificados (C) con 15 o más años de escolaridad.

El mercado de trabajo se dividió en siete sectores económicos o segmentos : industria manufacturera; construcción; electricidad, gas y agua potable; comercio, restaurantes y hoteles; transporte, almacenamiento y comunicaciones; servicios financieros, seguros, actividades inmobiliarias y de alquiler; y servicios comunales, sociales y personales.

A partir de los criterios establecidos, la muestra total abarcó 18.471 .768 observaciones para el año 1992 y 28.282.113 en el año $2014^{2}$. En la siguiente sección se presentan los resultados más relevantes sobre la composición del mercado laboral en México y las regiones.

\subsection{Regionalización}

En la literatura es posible encontrar tantas divisiones regionales como criterios e intereses se emplean para tal propósito. Con el fin de utilizar una regionalización que alcance a reflejar las dinámicas actuales de exposición al comercio exterior en México, se retoma la propuesta de Aguilera y Castro (2016), la cual consiste en una regionalización de tipo económico que agrupa a los Estados de la república mexicana (unidades político- administrativas subnacionales), según su grado de apertura comercial (ver gráfico 1).

Para ello, Aguilera y Castro (2016) recurrieron a técnicas de análisis regional, con la finalidad de conocer el grado de especialización y autosuficiencia de cada entidad mexicana e incluyeron variables que reflejan los vínculos con el comercio exterior, tales como: participación de las industrias manufactureras en el producto interno bruto (PIB) estatal, valor agregado censal bruto per cápita del sector manufacturero, porcentaje del empleo en las manufacturas sobre el total de empleo estatal, flujos de inversión extranjera directa (IED) y distancia a los Estados Unidos. Cabe señalar que el enfoque manufacturero responde a su relevancia en la dinámica del

Los autores se encuentran en disposición de suministrar la base de datos usada a cualquier investigador que la solicite, comunicándose al autor de correspondencia: david.castro@uadec.edu.mx 
sector externo de México, caracterizado por presentar concentración geográfica y sectorial $^{3}$. De lo anterior, se integraron tres regiones representadas en el gráfico 1.

\section{Gráfico 1. México. Regionalización en función del grado de exposición a la apertura comercial}

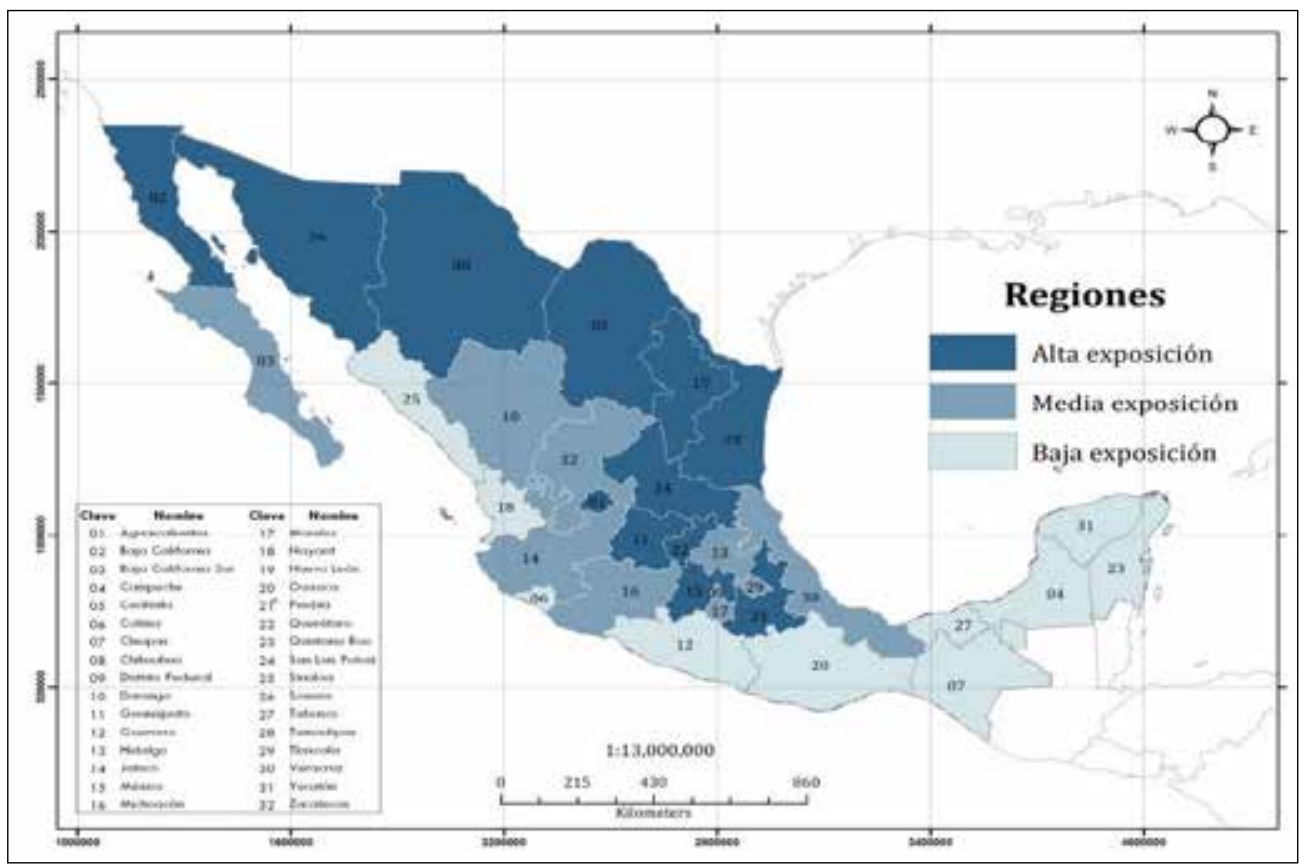

Fuente: elaboración propia con base en la propuesta de Aguilera y Castro (2016)

\section{TENDENCIAS DEL MERCADO DE TRABAJO REGIONAL EN MÉXICO}

La consolidación del proceso de apertura comercial en México, a raíz de la entrada en vigor del TLCAN, creó expectativas de crecientes flujos de IED y volúmenes de exportación que, a su vez, tendrían efectos directos en la generación de empleo, mejoras en el nivel del salario real y disminución de la migración.

Un examen de los componentes de la población económicamente activa (PEA) confirmó incremento generalizado de las tasas de desempleo (TD), en los años 1992 y 2014 (ver tabla 1). Así, las mayores tasas de desempleo impactan a los trabajadores de todas las regiones y niveles de calificación, aunque en mayor magnitud a los hombres, lo que llevó a una mayor convergencia en las TD por sexo.

3 En el 2015, el sector manufacturero contribuyó con más del 86 por ciento de las exportaciones totales de México, en tanto que, Estados Unidos se ha mantenido como su principal destino y socio comercial. En el año referido, se dirigieron más del 80 por ciento del total de las exportaciones mexicanas hacia el país vecino del norte, y cerca del 49 por ciento del total de importaciones procedieron de dicha nación. 
En el caso de las regiones de alta y media exposición, la TD llegó a 6,0 y 6,8\% en el 2014. En tiempos de crisis mundial, el comercio internacional es uno de los principales canales de transmisión hacia los mercados, reflejado en pérdidas de empleo en los sectores exportadores. En este contexto, es importante recordar que durante el período referido se originaron dos recesiones: la primera en 2001 propició una desaceleración del comercio exterior e impactó de forma negativa sobre las fuentes de generación de ocupaciones, en tanto que la crisis financiera de 2008 tuvo efectos adversos sobre la economía mexicana por su dependencia hacia los Estados Unidos, lo que motivó un deterioro de la actividad industrial y acentuó la informalidad y el autoempleo (Villarreal, 2010). En adición, el aumento de importaciones de insumos provenientes de China y otros países asiáticos ha reducido el crecimiento de las exportaciones mexicanas en el mercado de Estados Unidos, posición desfavorable para el mercado laboral.

Tabla 1. México. Tasa de desempleo regional por nivel de calificación, 1992 y 2014

\begin{tabular}{|c|c|c|c|c|c|c|c|}
\hline & \multicolumn{2}{|c|}{ Alta exposición } & \multicolumn{2}{|c|}{ Media exposición } & \multicolumn{2}{|c|}{ Baja exposición } \\
\hline & & 1992 & 2014 & 1992 & 2014 & 1992 & 2014 \\
\hline \multicolumn{2}{|l|}{ Total } & 3,7 & 6,0 & 4,1 & 6,8 & 2,7 & 5,3 \\
\hline \multirow[t]{3}{*}{ Hombres } & Calificado & 2,4 & 5,2 & 2,6 & 7,6 & 1,4 & 4,9 \\
\hline & Semi Calificado & 4,4 & 7,6 & 4,4 & 8,6 & 3,1 & 5,9 \\
\hline & No Calificado & 3,1 & 5,8 & 3,7 & 6,1 & 2,2 & 4,7 \\
\hline \multirow[t]{3}{*}{ Mujeres } & Calificado & 3,8 & 5,4 & 5,7 & 7,3 & 3,9 & 5,7 \\
\hline & Semi Calificado & 6,1 & 7,1 & 6,6 & 6,9 & 4,5 & 6,6 \\
\hline & No Calificado & 4,1 & 5,1 & 3,5 & 4,9 & 2,6 & 4,6 \\
\hline
\end{tabular}

Fuente: elaboración propia con datos del INEGI, ENEU 1992 y ENOE 2014, tercer trimestre

En la región de baja exposición el empleo se mantuvo superior, en comparación con el resto de las regiones (ver tabla 1). A razón de que México no cuenta con un seguro de desempleo, esto denotaría la necesidad de los habitantes por mantenerse ocupados para recibir algún ingreso y evitar la situación de pobreza. Por otro lado, la TD se incrementó en 2014 (5,3\%); pues, aunque la especialización económica de los Estados (división político administrativa subnacional) que integran esta región no es manufacturera, se han visto perjudicados a través de la caída de las remesas internacionales, segunda fuente de divisas más alta de México después del petróleo, y por las pérdidas en el sector turismo a raíz de las diversas catástrofes naturales y oleadas de violencia. 
Al comparar la estructura del empleo intra y entre regiones, los datos de la tabla 2 corroboran que, si bien el trabajo menos calificado exhibió mayor demanda en la región de alta exposición entre los años 1992 y 2014 (65,1 y 48,5\% hombres, y 55,4 y $44,2 \%$ mujeres), creció la intervención del trabajo calificado en el empleo regional, debido a un aumento en la participación de los hombres (7.8 puntos porcentuales) y las mujeres (13.4 puntos porcentuales).

En relación con el grupo de hombres semi-calificados, la información revela una tendencia progresiva de ocupación. En 2014 su participación fue superior al 29\% en las regiones de media y baja exposición, mientras que la intervención de las mujeres semi calificadas superó el 30\% (ver tabla 2). Estas cifras podrían sugerir efectos favorables producidos por el conjunto de reformas de ley aprobadas desde el 2012, que buscan impulsar la fuerza laboral juvenil y femenina al sector formal. En síntesis, los datos permiten inferir que, aunque la demanda total continúa intensiva en mano de obra no calificada, se observa una creciente ocupación de trabajadores semi calificados y calificados.

Tabla 2. México. Empleo regional según nivel de calificación laboral, 1992 y 2014 (Distribución porcentual)

\begin{tabular}{|l|cccccc|}
\hline \multirow{2}{*}{ Hombres } & \multicolumn{2}{|c}{ Alta exposición } & \multicolumn{2}{c}{ Media exposición } & \multicolumn{2}{c|}{ Baja exposición } \\
Calificados & 1992 & 2014 & 1992 & 2014 & 1992 & 2014 \\
Semi calificados & & & & & & \\
No calificados & 15,2 & 23 & 18,6 & 22,5 & 17,1 & 24,9 \\
Total & 19,7 & 28,5 & 20,2 & 29,4 & 22,1 & 29 \\
Mujeres & 65,1 & 48,5 & 61,2 & 48,1 & 60,8 & 46,1 \\
Calificados & 100 & 100 & 100 & 100 & 100 & 100 \\
Semi calificados & 13,6 & 27 & 15,1 & 25,4 & 14,1 & 28,1 \\
No calificados & 31 & 28,8 & 30,5 & 30,5 & 32,3 & 30,4 \\
Total & 55,4 & 44,2 & 54,4 & 44,1 & 53,5 & 41,5 \\
\hline
\end{tabular}

Fuente: elaboración propia con datos del INEGI, ENEU 1992 y ENOE 2014, tercer trimestre

\subsection{Evolución de los ingresos salariales regionales}

Pese a que México es uno de los países con menores tasas de desempleo entre los miembros de la OCDE, se ha puesto en evidencia que la calidad del ingreso en 
México es la más baja de toda la Organización, lo que atribuye a que el país cuente con un ingreso promedio reducido (OCDE, 2014). En el 2013 se colocó como la segunda nación miembro con el menor salario mínimo, justo por encima de la República Checa, situación alarmante ya que, según datos del INEGI, en el 2014 más de 10 millones de trabajadores que representan cerca de la cuarta parte de la población ocupada en el país recibieron hasta un salario mínimo.

El análisis del salario real que se resume en la tabla 3 muestra que, previo a la entrada en vigor del TLCAN y hasta el año 2014, las regiones de mayor exposición ofrecieron ingresos laborales superiores. No obstante, las remuneraciones intrarregionales registraron un significativo descenso entre los años incluidos en el análisis, de 21,4 y $11,8 \%$ en las regiones de media y alta exposición.

Tabla 3. México. Media del salario real por hora según grupos demográficos, 1992 y 2014 (pesos mexicanos de 2014*

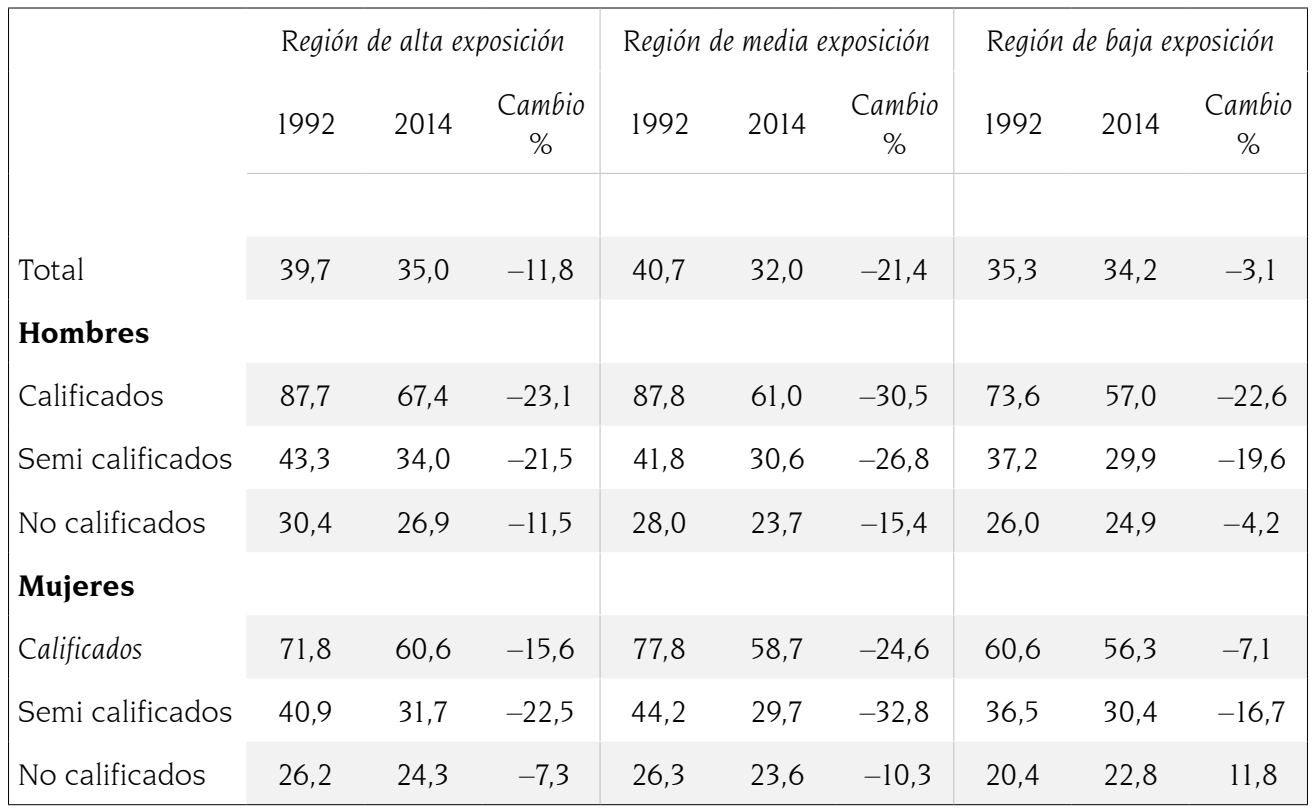

Nota: * En agosto de 2014 el tipo de cambio peso/dólar de Estados Unidos era: 13,12.

Fuente: elaboración propia con datos del INEGI, ENEU 1992 y ENOE 2014, tercer trimestre.

El análisis por grupos demográficos revela una relación positiva entre la calificación de los individuos y las remuneraciones que perciben, aunado a un detrimento generalizado de los niveles salariales que afectó especialmente a la mano de obra calificada y semi calificada de ambos sexos. Por otro lado, los grupos de trabaja- 
dores no calificados fueron los menos perjudicados con la reducción salarial; incluso, en la región de baja exposición el salario medio por hora de las mujeres no calificadas se incrementó en 11,8\% de 1992 a 2014 (ver tabla 3). Lo anterior podría derivarse de la política de salarios mínimos que establece límites para prevenir que decrezcan los sueldos de la parte baja de la distribución y como mecanismo para reducir la pobreza (Coronel, 2016).

Hasta ahora, el comportamiento de los salarios reales hace prever una disminución de las brechas salariales por sexo y entre grupos según el nivel de calificación, producto de un detrimento en las remuneraciones de los trabajadores más calificados, situación que se presenta con mayor intensidad en las regiones más expuestas a la apertura comercial. El deterioro constante del salario tiene un impacto desfavorable en el bienestar y las condiciones de vida de los mexicanos, e indica un menoscabo en la demanda agregada de la economía. En lugar de impulsar la mejora del ingreso laboral que promueva empleos formales en el país, se oferta mano de obra barata y en condiciones de flexibilidad como mayores ventajas competitivas del país.

\section{DESIGUALDAD SALARIAL EN LAS REGIONES DE MÉXICO}

Con la intención de examinar la evolución de la distribución del ingreso salarial en las regiones de México, se estimó el coeficiente de Gini a partir de los datos procedentes de la ENEU y ENOE. Los resultados que se resumen en el gráfico 2 indican que en 1992 existía una mayor inequidad en las regiones de media y baja exposición $(0,416$ y 0,410). Caso contrario se observa en la región de alta exposición $(0,397)$, lo cual podría sugerir un efecto positivo de la apertura comercial que había experimentado el país hasta ese momento, y mejores condiciones salariales por el crecimiento de los sectores exportadores.

En el año 2014 se presentó una reducción generalizada de las disparidades, donde la región de baja exposición exhibió la mayor desigualdad en el ingreso laboral, con un coeficiente de Gini de 0,393; en la región de alta exposición fue de 0,357 y de 0,348 en la de media exposición. En paralelo, los datos reflejan que en largo plazo se amplió la brecha salarial entre las regiones más y menos expuestas a la apertura (ver gráfica 1). Cabe señalar que, aunque la tendencia de las disparidades ha sido decreciente, de acuerdo con OCDE (2015) México continúa como uno de los países miembros con mayor desigualdad salarial solo por debajo de Chile. Sus cifras revelan que, en el 2012 el salario medio del 10\% más rico era 30,5 veces mayor que el $10 \%$ más pobre. 
Gráfico 2. México. Evolución de la desigualdad salarial por regiones, 1992 y 2014 (Coeficiente de Gini)

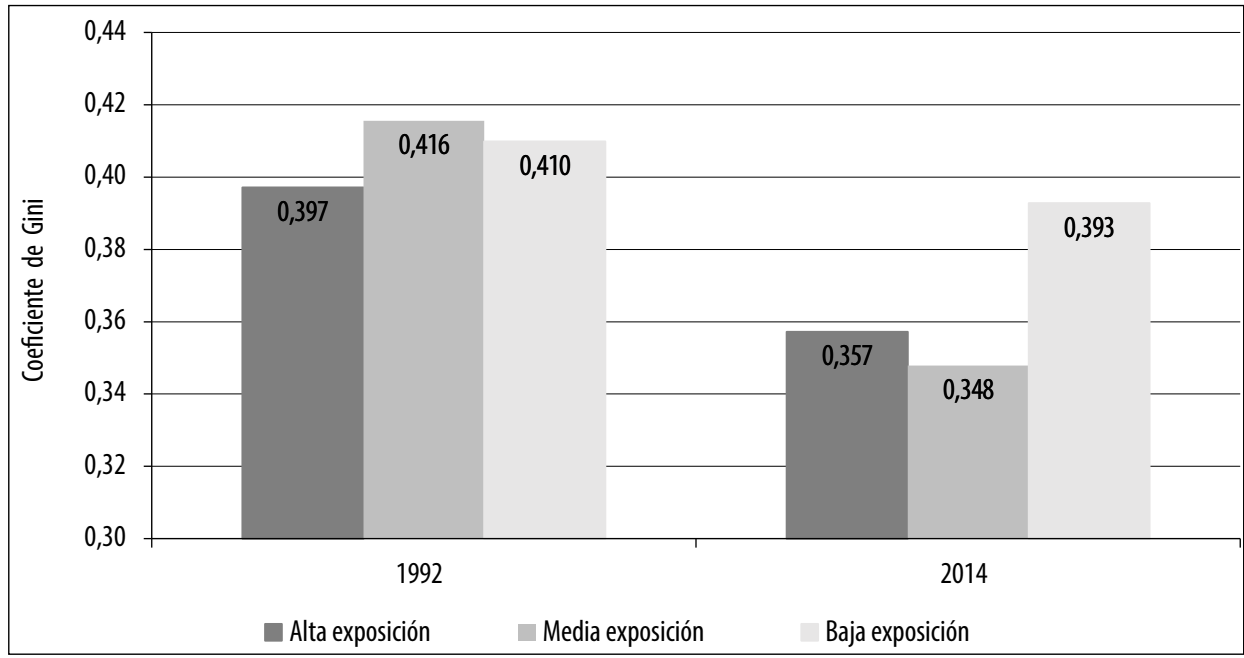

Fuente: elaboración propia con datos del INEGI, ENEU 1992 y ENOE 2014. Tercer trimestre

Con el fin de desagregar la desigualdad salarial total por grupos de trabajadores según su nivel de calificación, se estimó el índice de Theil que se presenta en la tabla 4. Destaca en primer término que el componente intra es la principal fuente para explicar la desigualdad de las agrupaciones calificadas, semicalificadas y no calificadas, con más del 69\% sobre el total.

Tabla 4. México. Descomposición del índice de Theil regional por nivel de calificación, 1992 y 2014

\begin{tabular}{|c|l|c|c|c|c|}
\hline Región & Componente & 1992 & $\%$ & 2014 & $\%$ \\
\hline \multirow{3}{*}{ Alta Exposición } & Total & 0,298 & 100 & 0,251 & 100 \\
& Intra & 0,212 & 71,1 & 0,181 & 72,2 \\
& Entre & 0,086 & 28,9 & 0,07 & 27,8 \\
& Total & 0,333 & 100 & 0,231 & 100 \\
Media Exposición & Intra & 0,231 & 69,4 & 0,160 & 69,3 \\
& Entre & 0,102 & 30,6 & 0,071 & 30,7 \\
& Total & 0,311 & 100 & 0,278 & 100 \\
Baja Exposición & Intra & 0,221 & 71,1 & 0,205 & 73,5 \\
& Entre & 0,090 & 28,9 & 0,074 & 26,5 \\
\hline
\end{tabular}

Fuente: elaboración propia con datos del INEGI, ENEU 1992 y ENOE 2014. Tercer trimestre 
Adicionalmente, las cifras sugieren que en el largo plazo la caída en el valor de la desigualdad provino de una reducción de ambos componentes, aunque en mayor proporción se contrajo la desigualdad interna (ver tabla 4). Esto indicaría menores brechas salariales en los conjuntos de trabajadores calificados, semi-calificados y no calificados, por lo que cobra importancia la necesidad de diseñar políticas de forma centralizada.

Hasta ahora, los resultados y la evidencia presentada parecen confirmar que posterior a la liberalización comercial en México, las regiones más expuestas a la apertura exhibieron menores niveles de desigualdad salarial y decreciente en el largo plazo, en relación con la región de baja exposición. Sin embargo, contrario a las predicciones teóricas, las tendencias no fueron producto de una mejora en las condiciones salariales de la mano de obra no calificada. Con el fin de identificar qué parte de las variaciones observadas en la distribución del ingreso puede ser atribuida a la liberalización comercial y cuál es ocasionada por otros factores, se aplicó la metodología de microsimulaciones.

\subsection{Liberalización comercial en México y su efecto a largo plazo sobre la desigualdad salarial regional}

La metodología de microsimulación involucra imputar la composición del mercado de trabajo de un año que refleje las condiciones económicas previas a la reforma comercial, sobre los datos de un año posterior a la misma. A razón de que se utilizan componentes aleatorios, cada ejercicio de simulación se replicó 100 veces $^{4}$ a través del método bootstrap, con el fin de establecer intervalos de confianza del 95\% en la estimación de los indicadores de desigualdad e identificar la significancia estadística de las variaciones, excepto en la simulación del cambio en la estructura de las remuneraciones que no involucra números al azar.

En la tabla 5 se presenta los valores de la desigualdad del ingreso laboral a largo plazo, este escenario indica el nivel de disparidad de los ingresos salariales si la composición del mercado laboral en 2014 no hubiera cambiado con respecto a la observada en 1992, previo a la firma del TLCAN. Se presentan los resultados de alterar de forma individual los parámetros del mercado laboral: tasa de participación, tasa de desempleo, estructura de empleo por sector económico, distribución de trabajadores por nivel de calificación e ingresos salariales.

Los ejercicios de simulación sugieren que, la distribución del empleo por nivel de calificación y el efecto ingreso, constituyen los factores relevantes que intervinieron en las variaciones de la desigualdad salarial en las tres regiones. Las cifras

\footnotetext{
4 Las simulaciones también se efectuaron con 50, 500 y 1000 repeticiones, pero los resultados fueron similares a los obtenidos con 100 .
} 
significativas en aminorar el coeficiente de Gini expresan que, a partir del proceso de liberalización comercial, la acentuación de la demanda de fuerza de trabajo calificada y semi calificada, así como la contracción del trabajo no calificado de ambos sexos, perturbaron de manera desfavorable las disparidades en el ingreso, ya que de haberse mantenido la misma estructura que prevalecía en 1992, la brecha salarial sería menor a la observada en el 2014. En la región de alta exposición se tendría un coeficiente de Gini de 0,345, es decir, 3,3\% más bajo; en la región de media exposición sería 4,8\% inferior, mientras en la región de baja exposición la reducción sería de $4,4 \%$ (ver tabla 5 ).

El efecto remuneración aumentó la desigualdad en lugar de disminuirla; esto implica que, si la estructura de ingresos laborales de México fuese la de 1992 se incrementarían las disparidades regionales (ver tabla 5), debido a que el coeficiente de Gini se ampliaría 4,8\% en la región de alta exposición, 6,4\% en la región de media exposición y 3,3\% en la región de baja exposición. Este comportamiento sugiere que la apertura comercial contribuyó a reducir la desigualdad en el largo plazo; sin embargo, en contraste con las predicciones teóricas, no fue producto de una mejora en el ingreso de los individuos asalariados, sino que deriva de un detrimento generalizado del salario real de los trabajadores, en particular sobre la mano de obra más calificada.

El efecto de la participación laboral resultó significativo en el sentido de contraer la desigualdad, 2,8\% en la región de media exposición. Esto significa que la liberalización comercial produjo un crecimiento de la misma en el largo plazo, y puede atribuirse a las secuelas que origina la migración. En las simulaciones realizadas por Bouillon (2000) y Ros y Bouillon (2002) en su estudio para México, las tasas de participación tuvieron el impacto más significativo dentro de las variables de demanda y oferta de trabajo, lo cual atribuyen a transformaciones demográficas como nivel educativo, número de hijos, composición del hogar e incrementos de la participación laboral de personas con Secundaria o mayor nivel educativo.

En otro orden de ideas, el desempleo y la estructura ocupacional sectorial no resultaron estadísticamente significativos. Una interpretación a lo primero es que las modificaciones en la TD de 1992 a 2014 fueron crecientes para los seis tipos de trabajadores considerados en el análisis; por consiguiente, podría contrarrestar el impacto sobre las diferencias en los niveles salariales. El hecho de que la composición en las ocupaciones por actividades económicas no sea estadísticamente significativa sugiere que, pese a las modificaciones sectoriales que se observaron después de la apertura, las remuneraciones por sectores no presentaron diferencias relevantes. También es posible especular que previo a la entrada en vigor del TLCAN, ya se había dado la reasignación de la mano de obra hacia las industrias exportadoras. 
Tabla 5. México. Micro-simulaciones: efectos individuales sobre la distribución del ingreso salarial regional, 1992 y 2014

\begin{tabular}{|c|c|c|c|c|}
\hline \multirow{2}{*}{$\begin{array}{l}\text { Regiones / Cambios } \\
\text { en los parámetros del } \\
\text { mercado laboral }\end{array}$} & \multirow[b]{2}{*}{ Ingreso real 2014} & \multirow{2}{*}{$\begin{array}{l}\text { Ingreso simulado (con } \\
\text { datos de 1992) }\end{array}$} & \multicolumn{2}{|c|}{ Cambios en el coeficiente de Gini } \\
\hline & & & Puntos & Porcentaje \\
\hline \multicolumn{5}{|c|}{ Región de alta exposición } \\
\hline \multirow[t]{2}{*}{ Fase 1} & 0,3572 & 0,3519 & $-0,0053$ & $-1,48 \%$ \\
\hline & & {$[0,3429 \quad 0,3595]$} & & \\
\hline \multirow[t]{2}{*}{ Fase 2} & 0,3572 & 0,3569 & $-0,0003$ & $-0,08 \%$ \\
\hline & & {$[0,3487 \quad 0,3649]$} & & \\
\hline \multirow[t]{2}{*}{ Fase 3} & 0,3572 & 0,3593 & 0,0021 & $0,59 \%$ \\
\hline & & {$[0,3515 \quad 0,3671]$} & & \\
\hline \multirow[t]{2}{*}{ Fase 4} & 0,3572 & 0,3454 & $-0,0118$ & $-3,30 \%$ \\
\hline & & {$[0,3387 \quad 0,3520]$} & & \\
\hline Fase 5 & 0,3572 & 0,3743 & 0,0171 & $4,79 \%$ \\
\hline \multicolumn{5}{|c|}{ Región de media exposición } \\
\hline \multirow{2}{*}{ Fase 1} & 0,3479 & 0,3382 & $-0,0097$ & $-2,79 \%$ \\
\hline & & {$[0,3263 \quad 0,3472]$} & & \\
\hline \multirow[t]{2}{*}{ Fase 2} & 0,3479 & 0,3478 & $-0,0001$ & $-0,03 \%$ \\
\hline & & {$[0,3365 \quad 0,3590]$} & & \\
\hline \multirow[t]{2}{*}{ Fase 3} & 0,3479 & 0,3454 & $-0,0025$ & $-0,72 \%$ \\
\hline & & {$[0,3340 \quad 0,3568]$} & & \\
\hline \multirow[t]{2}{*}{ Fase 4} & 0,3479 & 0,3312 & $-0,0167$ & $-4,80 \%$ \\
\hline & & {$[0,3211 \quad 0,3413]$} & & \\
\hline Fase 5 & 0,3479 & 0,3703 & 0,0224 & $6,44 \%$ \\
\hline \multicolumn{5}{|c|}{ Región de baja exposición } \\
\hline \multirow[t]{2}{*}{ Fase 1} & 0,3928 & 0,391 & $-0,0018$ & $-0,46 \%$ \\
\hline & & {$[0,3842 \quad 0,3976]$} & & \\
\hline \multirow[t]{2}{*}{ Fase 2} & 0,3928 & 0,3925 & $-0,0003$ & $-0,08 \%$ \\
\hline & & {$[0,3859 \quad 0,3989]$} & & \\
\hline \multirow[t]{2}{*}{ Fase 3} & 0,3928 & 0,3936 & 0,0008 & $0,20 \%$ \\
\hline & & {$[0,3873 \quad 0,3998]$} & & \\
\hline \multirow[t]{2}{*}{ Fase 4} & 0,3928 & 0,3754 & $-0,0174$ & $-4,43 \%$ \\
\hline & & {$[0,3691 \quad 0,3816]$} & & \\
\hline Fase 5 & 0,3928 & 0,4064 & 0,0136 & $3,46 \%$ \\
\hline
\end{tabular}

Notas:

a) Cada fase corresponde a valores en caso de cambio de 1. Tasa de participación; 2. Tasa de desempleo; 3. Estructura de empleo (sectores); 4. Estructura de empleo (calificación), y 5. Estructura de remuneración.

b) El ingreso corresponde al salario real por hora expresado en pesos mexicanos del 2014.

c) Los valores en corchetes representan los intervalos de confianza al 95\%. La fase 5 no muestra intervalos de confianza porque se aplica la estructura salarial de 1992 sin generar cifras aleatorias.

d) Los valores en negrita y resaltados indican diferencia estadísticamente significativa.

Fuente: elaboración propia con datos del INEGI, ENEU 1992 y ENOE 2014. Tercer trimestre. 
Finalmente, es importante señalar que los resultados aquí presentados están en armonía con el informe de la OIT (2015), donde se establece que en muchos países las disparidades de ingreso comienzan en el mercado de trabajo, y son la distribución de los salarios y el empleo remunerado los factores predominantes en las tendencias de la desigualdad, por lo que asienta la importancia de las instituciones y las políticas del mercado laboral, la negociación colectiva y las políticas fiscales, con el fin de incidir en una redistribución de los ingresos más favorable.

\section{CONCLUSIONES}

Con base en las predicciones de la teoría convencional de comercio internacional, en esta investigación se examina la desigualdad salarial entre trabajadores con distintas dotaciones de calificación, en los años 1992 y 2014. El análisis se realiza considerando una clasificación regional de México y a partir de los datos de la ENEU y ENOE.

Los hallazgos de esta investigación suponen una contribución al análisis de la desigualdad salarial regional, dado que considera aspectos como el proceso de apertura comercial, un período de estudio amplio y reciente, diversas fuentes de información, el uso de una metodología adaptada a una escala regional, y la incorporación de un método de clasificación de calificación de mano de obra no reportado en el caso mexicano.

Los resultados muestran que posterior a la liberalización comercial en México, las regiones con mayor exposición a la apertura mantuvieron inferiores niveles de desigualdad salarial (0,357 y 0,348 alta y media exposición), en relación con la región menos expuesta $(0,393)$. Sin embargo, esto derivó de un detrimento generalizado del salario real que afectó a la mano de obra más calificada, tanto del sexo femenino como masculino.

La comparación entre los años 1992 y 2014 mediante la metodología de microsimulaciones ofreció un balance de los impactos distributivos que tuvieron lugar en los años estudiados. La evidencia de la simulación indica que, si el proceso de apertura no se hubiera implementado, los índices de disparidad serían inferiores a los observados en el 2014. El empleo por nivel de calificación de la población asalariada y el nivel de ingreso fueron las principales fuentes de cambio de la desigualdad de los ingresos laborales.

A partir de lo dicho hasta ahora se puede establecer que, contrario a las predicciones del teorema Stolper-Samuelson, la liberalización comercial no contribuyó a reducir las disparidades de ingreso a largo plazo; sin embargo, las regiones con mayor exposición presentaron mejores condiciones salariales. 
Finalmente, es relevante señalar que el documento también pone de manifiesto el hecho de que en el comportamiento de los salarios y la desigualdad salarial regional, pudieron influir factores adicionales a la apertura comercial, como puede ser el cambio tecnológico, factores de oferta, institucionales e incluso coyunturales; sin embargo, no se puede soslayar la relevancia de la apertura comercial sobre el comportamiento de los salarios y la desigualdad, al igual que la necesidad de mayor investigación sobre el tema.

\section{BIBLIOGRAFÍA}

Acemoglu, D. (1998). Changes in unemployment and wage inequality: an alternative theory and some evidence. En: National Bureau of Economic Research, No. w6658, 45p.

Aguilera, A. y Castro, D. (2016). La dinámica regional y el proceso de apertura comercial en México. Capítulo 2, p.47-86. En: Castro Lugo, David y Rodríguez Pérez, Reyna E. (eds). Mercado laboral en México: situación y desafíos. $1^{a}$ edición, Universidad Autónoma de Coahuila, Ediciones de Laurel, México, 239p.

Aguilera, A. y Castro, D. (2017). Calificación laboral y desigualdad salarial; un ejercicio metodológico por conglomerados. En: Working Paper, Universidad Autónoma de Coahuila, Version: 1. DOI:10.13140/RG.2.1.4028.6485.

Airola, J. y Juhn, C. (2005). Wage inequality in post-reform Mexico. En: IZA Discussion Paper, No. 1525, 48p.

Almeida Dos Reis, J. y Paes De Barros, R. (1991). Wage inequality and the distribution of education: A study of the evolution of regional differences in inequality in metropolitan Brazil. En: Journal of Development Economics, Vol. 36, No. 1, p. 117-143.

Blecker, R. (2010). Comercio, empleo y distribución: efectos de la integración regional y global. Capítulo 5, p. 175-214. En: Lustig, Nora (coord.). Los grandes problemas de México. Tomo 9. Crecimiento económico y equidad. El Colegio de Mexico, México, 324p.

Borraz, F. y López-Córdova, J. (2007). Has Globalization Deepened Income Inequality in Mexico? En: Global Economy Journal, Vol. 7, No. 1, p.1-55.

Bouillon, C. (2000). Inequality and Mexico's labor market after trade reform. En: Inter-American Development Bank, No. 53718, 34p.

Burgos, B. y Mungaray, A. (2008). Apertura externa, inequidad salarial y calificación laboral en MéxiCo, 1984-2002. En: Problemas del Desarrollo, Vol. 39, No. 152, p. 87-111.

Camberos, M.; Huesca Reynoso, L. y Castro, D. (2013). Cambio tecnológico y diferencial salarial en las regiones de México: un análisis de datos de panel para el sector servicios. En: Estudios fronterizos, Vol. 14, No. 28, p- 187-211.

Cañonero, G. y Werner, A. (2002). Salarios relativos y liberación del comercio en México. En: El Trimestre Económico, Vol. 69, No. 273(1), p. 123-142.

Castro, D. y Huesca, L. (2007). Desigualdad salarial en México: una revisión. En: Papeles de Población, Vol. 13, No. 54, p. 225-264. 
Chiquiar, D. (2008). Globalization, Regional Wage Differentials and the Stolper-Samuelson Theorem: Evidence from Mexico. En: Journal of International Economics, Vol. 74, No. 1, p. 70-93.

Coronel, J. (2016). Ingresos laborales y tiempos en el trabajo en Antioquia y el Área metropolitana del Valle de Aburrá 2012-2014. En: Semestre Económico, Vol. 19, No. 39, p. 61-89.

Cortez, W. (2004). Un análisis de los factores detrás de los cambios en la inequidad salarial en México. En: Carta Económica Regional, No. 88, p. 50-60.

De La Mora, L.M. (2015). El comercio exterior como palanca del crecimiento económico y desarrollo de México. En: Revista de Comercio Exterior, No. 4 (Nueva época), octubre-diciembre, p. 1-6.

Ganuza, E.; De Barros, R. y Vos, R. (2002). Labour market adjustment, poverty and inequality during liberalisation. Capítulo 2, p. 54-89. En: Rob Vos, Lance Taylor y Ricardo Paes de Barros (Ed.). Economic Liberalisation, Distribution and Poverty: Latin America in the 1990s. Edward Elgar Publishing y United Nations Development Programme (UNDP), 440p.

Garduño, R. y Baylis, K. (2012). Effect of Tariff Liberalization on Mexico's Income Distribution in the presence of Migration. Presentación para Agricultural and Applied Economics Association, No. 124740, 23p.

Hanson, G. (2007). Globalization, Labor Income, and Poverty in Mexico, p. 417-454. En: Ann Harrison (editor). Globalization and poverty. University of Chicago Press, 536p.

López, N. G. (2015). The effect of trade and FDI on inter-industry wage differentials: The case of Mexico. En: The North American Journal of Economics and Finance, Vol. 34, noviembre, p. 381-397. https://doi.org/10.1016/j.najef.2015.09.006

Luyando, J.R. (2013). Desigualdad salarial antes y durante la apertura comercial mexicana. En: Revista Lebret, No. 5, p. 29-50. DOI: http://dx.doi.org/10.15332/rl.v0i5.812

OCDE (2014). Employment Outlook 2014. Organización para la Cooperación y el Desarrollo Económico, 292p.

OCDE (2015). Employment Outlook 2015. Organización para la Cooperación y el Desarrollo Económico, 296p.

OIT (2015). Informe mundial sobre salarios 2014/2015: Salarios y desigualdad de ingresos. Organización Internacional del Trabajo (OIT), febrero, 144p.

Pérez, R. (2014). Determinantes de la desigualdad salarial en el Estado de México, 2000-2009. CIEPS, México, 114p.

Ramírez, C. (2004). Desigualdad salarial y desplazamientos de la demanda calificada en México, 19931999. En: El Trimestre Económico, Vol. 71, No. 283(3), julio-septiembre, p. 625-680.

Robertson, R. (2004). Relative prices and wage inequality: evidence from Mexico. En: Journal of International Economics, Vol. 64, No. 2, p. 387-409.

Ros, J. y Bouillon, C. (2002). Mexico: trade liberalization, growth, inequality and poverty. Capítulo 9 , p. 347-390. En: Rob Vos, Lance Taylor y Ricardo Paes de Barros (Ed.). Economic Liberalisation, Distribution and Poverty: Latin America in the 1990s. Edward Elgar Publishing y United Nations Development Programme (UNDP), 440p.

Shorrocks, F. (1980). The class of additively decomposable inequality measures. En: Econometrica: Journal of the Econometric Society, Vol. 48, No. 3, abril, p. 613-625. 
Stolper, W. y Samuelson, P. (1941). Protection and Real Wages. En: The Review of Economic Studies, Vol. 9, No.1, p. 58-73.

Tello, C. y Ramos, R. (2012). Wage inequality and economic growth in Mexican regions. En: Investigaciones Regionales, No. 24, p. 115-152.

Villarreal, D. (2010). Efectos regionales del cambio de modelo de desarrollo económico de México, 1980-2006. Capítulo 6, p. 163-198. En: José Flores Salgado (coord.) Crecimiento y desarrollo económico de México. Universidad Autónoma Metropolitana, Unidad Xochimilco, División de Ciencias Sociales y Humanidades, 273p. 